SANUS. 2020;(16):1-24

\title{
Proceso de enfermería a usuaria con diagnóstico de Neumonía por SARS-CoV-2
}

\author{
Dayanira Ariadna Diaz-Correa ${ }^{1}$; Sandra Lidia Peralta-Peñaa ${ }^{2 *}$ María Rubí Vargas ${ }^{3}$
}

\begin{abstract}
RESUMEN
Introducción: Tras la pandemia por el virus SARS-CoV-2 la participación de la enfermería profesional a sido parte medular del éxito en la atención de usuarios diagnosticados con COVID-19 debido a la alta tasa de mortalidad a nivel mundial. El uso de metodologías propias de enfermería marca la pauta para un mejor razonamiento clínico asegurando así una precisión diagnóstica y terapéutica. Objetivo: Presentar una propuesta de cuidado enfermero siguiendo métodos y recursos propios de la disciplina enfermera. Metodología: Se empleó el proceso de enfermería como método de intervención, las taxonomías de la NANDA, NOC y NIC. La precisión de los diagnósticos se estimó a través de la Escala de Precisión de Diagnósticos de Enfermería de Lunney y la jerarquización de diagnósticos con la red de razonamiento clínico del modelo AREA. Presentación del caso: Usuario femenino de 67 años diagnosticado con Neumonía por SARS-CoV-2 que ingresa al servicio de Terapia Intensiva tras doce días de hospitalización en Medicina Interna y siete días de apoyo ventilatorio invasivo con sedoanalgesia. Conclusiones: La aplicación del proceso de enfermería y recursos complementarios permitieron que se obtuvieran diagnósticos más precisos, su jerarquización y el desarrollo del juicio terapéutico al intervenir.
\end{abstract}

Descriptores de salud: Proceso de enfermería; Planificación de atención al paciente; Infecciones por coronavirus (DeCS; BIREME).

\footnotetext{
${ }^{1}$ Enfermera Especialista en Cuidados Intensivos. Hospital San José, Hermosillo. Correo electrónico:

dayaniradiaz@hotmail.com ORCID-iD: 0000-0002-6521-2608.

${ }^{2}$ Doctor en Ciencias de Enfermería. Departamento de Enfermería de la Universidad de Sonora. Hermosillo, Sonora, México.

ORCID-iD 0000-0001-8534-2250.

${ }^{3}$ Doctor en Ciencias de Enfermería. Departamento de Enfermería de la Universidad de Sonora.

ORCID-iD 0000-0002-1075-5576.
}

*Autor para correspondencia: sandra.peralta@unison.mx 


\title{
Nursing process used in a user with pneumonia due to SARS-CoV-2 diagnosis
}

\begin{abstract}
Introduction: After the pandemic due to SARS-CoV-2, the participation of the professional nursing has been a core part of the success in the healthcare of users who have been diagnosed with COVID-19, due to a high mortality rate worldwide. The use of internal nursing methodologies makes the difference for a better clinical reasoning, thus assuring a diagnostic and therapeutic accuracy. Objective: Present a proposal of nursing care following methods and resources of the own nursing discipline. Methodology: The nursing process as intervention method and the taxonomies of NANDA, NOC, and NIC were used. The accuracy of the diagnosis was estimated through the Lunney's Accuracy of Nursing Diagnoses Scale and the prioritization of diagnosis with the reasoning network of the AREA model. Presentation of the case: Female user of 67 years of age diagnosed with pneumonia due to SARS-CoV-2 who entered to the intensive therapy unit after twelve days of hospitalization in internal medicine and seven days of invasive ventilation support with sedoanalgesia. Conclusions: The applications of the nursing process and complementary resources allowed obtaining more precise diagnoses, their prioritization, and the development of the development of the therapeutic judgment during intervention.
\end{abstract}

Health descriptors: Nursing process; planning the healthcare to the patient; coronavirus infection (DeCS; BIREME). 


\title{
Processo de enfermagem utilizado em um usuário com pneumonia devido ao diagnóstico de SARS-CoV-2
}

\begin{abstract}
ABSTRATO
Introdução: Após a pandemia devido à SARS-CoV-2, a participação do profissional de enfermagem tem sido uma parte essencial do sucesso na assistência à saúde de usuários que foram diagnosticados com COVID-19, devido a uma alta taxa de mortalidade em todo o mundo. A utilização de metodologias internas de enfermagem faz a diferença para um melhor raciocínio clínico, garantindo assim uma acurácia diagnóstica e terapêutica. Objetivo: Apresentar uma proposta de cuidado de enfermagem seguindo métodos e recursos da própria disciplina de enfermagem. Metodologia: Utilizou-se o processo de enfermagem como método de intervenção e as taxonomias NANDA, NOC e NIC. A acurácia do diagnóstico foi estimada por meio da Escala de Precisão de Diagnósticos de Enfermagem de Lunney e a priorização do diagnóstico com a rede de raciocínio do modelo AREA. Presentation of the case: Female user of 67 years of age diagnosed with pneumonia due to SARS-CoV-2 who enters to the intensive therapy unit after twelve days of hospitalization in internal medicine and seven days of invasive ventilation support with sedoanalgesia. Conclusions: The applications of the nursing process and complementary resources allowed obtaining more precise diagnoses, their prioritization, and the development of the development of the therapeutic judgment during intervention.
\end{abstract}

Descritores de saúde. Processo de enfermagem; planejar o cuidado ao paciente; contágio do coronavírus (DeCS; BIREME). 


\section{INTRODUCCIÓN}

El 11 de marzo del 2020, la Organización Mundial de la Salud (OMS), declaró la presencia de la pandemia por el virus SARS-CoV-2 o COVID-19. Hasta el día 24 de octubre a nivel nacional se han confirmado 886,800 casos totales y 88,743 defunciones totales por COVID-19. En Sonora a la misma fecha se registran 37,338 casos acumulados, colocándose entre las 10 primeras entidades del país que acumulan mayor número de casos (2). Los principales factores que aumentan la severidad del COVID-19 son las enfermedades crónico-degenerativas como diabetes mellitus, hipertensión arterial, sobrepeso y obesidad; el estado de Sonora ocupa los primeros lugares en la prevalencia nacional de estas condiciones ${ }^{(3)}$.

Hoy en día, la enfermería cobra especial relevancia, pues como integrante del equipo multidisciplinario de salud, es quien proporciona permanentemente los cuidados sustentados en una base científica con una visión integral, que la hace líder para afrontar todas las situaciones con ética y diferentes enfoques: administrativo, investigativo, docente y asistencial, tanto en el ámbito clínico como el comunitario (4).

En el caso de los pacientes con COVID-19, los cuidados de enfermería específicamente en el paciente crítico, tienen como objetivo tratar los diagnósticos enfermeros, identificar tempranamente complicaciones y evitar la transmisión en la población de riesgo ${ }^{(5)}$. Además, enfermería es el principal apoyo para los usuarios, pues son aislados de manera rotunda de su familia y entorno, generando sentimientos como temor y ansiedad ante el riesgo de muerte. El presente estudio de caso tiene como propósito presentar una propuesta de cuidado de enfermería a una usuaria con diagnóstico médico de Neumonía por SARS-CoV2, utilizando como método de intervención el proceso de enfermería, los lenguajes estandarizados de enfermería y herramientas como el modelo AREA y la Escala de
Precisión de Diagnósticos de Enfermería de Lunney.

\section{METODOLOGÍA}

El cuidado de enfermería a la señora CMM se implementó en una institución privada de tercer nivel de atención del noreste de México durante 45 días. Se empleó el proceso de enfermería como método de intervención ${ }^{(6-8)}$. Para la valoración de enfermería se utilizó el marco de valoración por Patrones Funcionales de Salud de Marjory Gordon (9,10); los diagnósticos, resultados e intervenciones se formularon haciendo uso del lenguaje estandarizado de enfermería: la taxonomía de la North American Nursing Diagnosis Association (NANDA) ${ }^{(11-14,18)}$, la taxonomía de la Nursing Outcomes Classification (NOC) $(15,16,18)$ y la taxonomía de la Nursing Interventions Classification (NIC) ${ }^{(13,17,18)}$. La precisión de los diagnósticos de enfermería se estimó a través de la Escala de Precisión de Diagnósticos de Enfermería (EPDE) de Lunney (19,20). La jerarquización de los diagnósticos se estableció mediante la red de razonamiento clínico del modelo Análisis de Resultado del Estado Actual (AREA) ${ }^{(21-23)}$. Se solicitó al usuario y su familia el consentimiento informado, dando garantía de la confidencialidad y anonimato de la información recabada.

\section{PRESENTACIÓN DEL CASO}

CMM, paciente femenino de 67 años, que acude a consulta el 27 de agosto por disnea de mínimos esfuerzos, fiebre, cefalea, tos productiva, mialgias, artralgias, dolor retro ocular y diarrea. Con apoyo de estudios sanguíneos y tomografía computarizada de tórax es diagnosticada con Neumonía por SARS-CoV2 por lo que se hospitaliza en el servicio de Medicina Interna. 
El 1 de septiembre muestra deterioro ventilatorio y neurológico, se inicia ventilación mecánica invasiva por tubo endotraqueal, se somete a sedoanalgesia con miorrelajación y se coloca en posición prona. Veinticuatro horas después, presenta inestabilidad hemodinámica y oliguria, se inician aminas vasopresoras e inotrópicos y con apoyo de un ecocardiograma transesofágico se identifica tromboembolia pulmonar y es sometida a trombectomía por medio de fibrinólisis intraarterial. También se coloca catéter Mahurkar para el inicio de terapia de reemplazo renal (hemodiálisis).

El 3 de septiembre, presenta edema de manera súbita en extremidades superiores, tórax y cuello; la tomografía muestra enfisema subcutáneo en tórax izquierdo, neumomediastino y aire libre subdiafragmático. Se realiza fasciotomía en región pectoral bilateral generando el escape instantáneo de aire y la disminución paulatina del enfisema subcutáneo. Ingresa al servicio de terapia intensiva el 8 del mismo mes, donde comienza con sangrado profuso en heridas de fasciotomía; hematología confirma coagulopatía; sin embargo, dado que el sangrado es local, se procede al cierre de heridas para hemostasia. El 20 de septiembre se realiza traqueotomía por la imposibilidad de un destete ventilatorio exitoso. Se realiza valoración de enfermería el día 29 de septiembre del 2020.

\section{Valoración de enfermería}

Patrón Mantenimiento-Percepción de la salud

CMM radica en Hermosillo, Sonora, es comerciante y ama de casa; vive en casa propia con los servicios básicos. Esquema de vacunación incompleto, sin especificar. Diagnosticada con hipotiroidismo hace 40 años, bajo tratamiento con levotiroxina $50 \mathrm{mcg}$ VO c/24 h; diabetes mellitus tipo 2 hace 10 años, controlada con linagliptina y metformina $2.5 / 1000 \mathrm{mg} \mathrm{VO} \mathrm{c} / 24 \mathrm{~h}$; hipertensión arterial sistémica de 5 años de evolución dependiente de telmisartán 20 mg VO c/24; con mal apego al tratamiento. Refiere alergia al ácido acetil salicílico. Niega el uso de sustancias psicoactivas.

Se observa la usuaria en mal estado general, con higiene deficiente. Tratamiento médico: metronidazol 500mg VO c/8h, omeprazol $40 \mathrm{mg}$ IV c/24h, budesonida con formoterol $160 / 4.5 \mathrm{mcg}$ inhalada c/12h, gluconato de calcio $1 \mathrm{gr} I V \mathrm{c} / 12 \mathrm{~h}$, clonixinato de lisina $100 \mathrm{mg}$ IV c/8h, enoxaparina $60 \mathrm{mg}$ SC c/24h, olanzapina $10 \mathrm{mg}$ VO c/24h, bromuro de ipratropio con acetilcisteína 250/250mg nebulizada c/8h y vancomicina $125 \mathrm{mg}$ VO c/6h. Cuenta con Nutrición parenteral N7 2000ml + 1 ámpula de multivitamínico + 1 gr de ácido ascórbico para $84.17 \mathrm{ml} / \mathrm{h} \mathrm{y}$ norepinefrina $16 \mathrm{mg}+$ sol. glucosada $5 \% 100 \mathrm{ml}$ $\mathrm{p} /$ dosis respuesta. Presenta alto riesgo de caídas con 13 puntos en la escala de Crichton. Patrón disfuncional.

\section{Patrón Nutricional-Metabólico}

Paciente normo térmico, IMC de $30 \mathrm{~kg} / \mathrm{m}^{2}$, piel deshidratada con turgencia disminuida y ligera palidez. Mucosa orofaríngea subhidratada, labios secos, halitosis, cuenta con 6 piezas dentales, presencia de sarro y caries en todas. Traqueostomía funcional, estoma con coloración rosada, sin fuga de secreciones ni infección. Heridas quirúrgicas de $5 \mathrm{~cm}$. en ambos pectorales con bordes bien afrontados, sin datos de infección. Tolera vía oral, pero evita comer por distención y peristaltismo aumentado; con tendencia a la hiperglucemia (Glucosa sérica de $173 \mathrm{mg} / \mathrm{dl}$ ). Presenta lesión por dependencia grado Il de tipo mixto ${ }^{(24)}$ en región sacro-coccígea e interglútea de $5 \mathrm{~cm}$ de diámetro y $2 \mathrm{~mm}$ de profundidad, cubierta con parche hidrocoloide; presenta hiperemia e inflamación en la piel de la periferia, con bordes engrosados; hay presencia de tejido macerado y esfacelar en los bordes. En el centro sus características cambian a una lesión por presión categoría III con pérdida total del grosor de la piel, también cuenta con tejido esfacelado y dos áreas de $.5 \mathrm{~cm}$ cada uno de tejido necrótico húmedo. Extremidades sin edema con 
medias compresivas. Ingreso total en 7 horas de $1270 \mathrm{ml}$. Los resultados de laboratorio muestran anemia $(\mathrm{Hb}: \quad 8.88 \mathrm{gr} / \mathrm{dl})$, leucopenia $\left(3.7110^{\wedge} 3 / \mathrm{ml}\right)$, linfopenia $\left(0.9810^{\wedge} 3 / \mathrm{ml}\right)$ e hiponatremia (Na: 134mg/dl). Patrón disfuncional.

\section{Patrón Eliminación}

Cuenta con sonda vesical de silicón tipo Foley \#16 $\mathrm{Fr}$, con uresis de $134.2 \mathrm{ml} / \mathrm{h}$, diuresis horaria de $1.45 \mathrm{ml} / \mathrm{kg} / \mathrm{h}$ y total de $940 \mathrm{ml}$ en el turno, densidad urinaria de 1020 . Tiene 15 días sin terapia de reemplazo renal por medio de hemodiálisis. Evacuaciones Bristol 6, color verdoso y fétidas con 3-5 evacuaciones al día (resultado positivo a clostridium difficile GDH + Toxina $A$ y $B$ en heces). Ruidos peristálticos hiperactivos de $8 x^{\prime}$. Abdomen con abundante panículo adiposo, distendido y timpánico. Pérdidas insensibles de $322 \mathrm{ml}$ en 7 horas $(92 \mathrm{~kg}$ x $0.5 \times 7$ horas), dando un balance hídrico de $42 \mathrm{ml}$. La química sanguínea muestra urea 57.8 $\mathrm{mg} / \mathrm{dl}$ y creatinina $0.7 \mathrm{mg} / \mathrm{dl}$ y tasa de filtrado glomerular $89.7 \mathrm{ml} / \mathrm{min} / 1.73 \mathrm{~m} 2$. Patrón disfuncional.

\section{Patrón Actividad-Ejercicio}

Signos vitales: FC 89x', TA 125/62mmHg, TAM $86 \mathrm{mmHg}, \mathrm{Fr} 36 x^{\prime}, \mathrm{SaO} 2$ de $100 \%$ con apoyo de mascarilla con pieza en $\mathrm{T}$ a $40 \%$ de $\mathrm{FiO} 2$ intermitente, con VM en modo espontáneo cada 3 horas PEEP $6 \mathrm{~cm} \mathrm{H} 2 \mathrm{O}$, PS 8 y $\mathrm{FiO} 2$ al 30\%. Campos pulmonares con estertores apicales bilaterales, tos productiva con secreciones de moderada cantidad, espesas y verdosas, de difícil expectoración. Con tendencia a la taquicardia, polipnea y disnea al mínimo esfuerzo. Catéter venoso central en subclavia izquierda $8.5 \mathrm{Fr}$ de 4 lúmenes, sin datos de infección a 28 días de instalación. Dependiente de norepinefrina a muy bajas dosis $(0.0056 \mathrm{mcg} / \mathrm{kg} / \mathrm{min})$, sin tolerar su retiro pues presenta disminución de PAM hasta $55 \mathrm{mmHg}$.

Extremidades íntegras, llenado capilar de 4s, capacidad de movilización incompleta ya que solo puede mover las manos y los ortejos de ambos pies. Fuerza disminuida e hiporreflexia en miembros torácicos y pélvicos (Grado 2 en Escala de Daniels). Actualmente se encuentra en sillón tipo reposet al que fue trasladada con total apoyo del personal de salud. La última gasometría arterial muestra acidosis metabólica medianamente compensada $(\mathrm{pH}$ 7.34, pCO2 $32.90 \mathrm{mmHg}$, pO2 $51 \mathrm{mmHg}$, Eb -8.2, HCO3 $17.8 \mathrm{mmol} / \mathrm{L}$, Dióxido de carbono $18.8 \mathrm{mmol} / \mathrm{L}$, spO2 83\%) y un Índice de Kirby de 196 $(\mathrm{PaO} 2=59 / \mathrm{FiO} 230 \% * 100)$, lo cual indica alteración en la ventilación/perfusión que requiere de mayor aporte de O2. Patrón disfuncional.

Patrón Sueño-Descanso

CMM comenta que su calidad de sueño es baja, tiene dificultad para conciliar el sueño, requiere de apoyo medicamentoso. Su sueño es interrumpido debido a los cuidados de enfermería, la mayor parte del día se siente cansada y con sueño, recientemente ha presentado cambios de humor y en ocasiones se niega a cooperar con el manejo. Patrón disfuncional.

\section{Patrón Cognitivo-Perceptual}

Se encuentra alerta, orientada en sus tres esferas, diámetro pupilar dentro de rangos normales y simétricos. Agudeza visual disminuida con antecedentes del uso de auxiliares externos desde hace 20 años por astigmatismo e hipermetropía. Su capacidad de habla actualmente se encuentra afectada por la traqueostomía, se comunica a través de señales, y del movimiento de labios y facies. Refiere dolor agudo de tipo somático con 8 según escala visual analógica (EVA) en glúteos por lesión por dependencia que disminuye con antiinflamatorios no esteroideos. Patrón disfuncional.

Patrón Autopercepción y Concepto de sí mismo 
CMM se considera una persona alegre, la percepción de su imagen corporal es negativa ya que se siente "demasiado gorda"; está conforme con su forma de ser y teme perder el habla y la movilidad física de manera permanente. Como consecuencia de la hospitalización y las complicaciones de su padecimiento, ha pasado por periodos de desmotivación y apatía. Patrón disfuncional.

\section{Patrón Rol-Relaciones}

La usuaria es casada, tiene tres hijos con los cuales lleva muy buena relación. CMM vive con su esposo e hija; el matrimonio es proveedor del hogar, por lo que le preocupa la disminución en la capacidad motriz. Sabe que cuenta con el apoyo de su familia y amigos. Patrón potencialmente disfuncional.

Patrón Sexualidad-Reproducción:

Menarquia a los 13 años, inicio de vida sexual activa a los 17; embarazos 3, partos 2 y cesárea 1 ; se realizó mamografía y examen cervicovaginal el año pasado sin reportar alteración. Inició la menopausia a los 50 años. A la exploración física sin datos de alteración. Patrón funcional.

Patrón Adaptación y Tolerancia al estrés

CMM refiere dificultades para adaptarse a las limitantes de su nueva situación, principalmente a la disminución de la capacidad motriz; la manera como canaliza su estrés y miedo es con periodos de ira, debido a la frustración que le genera ser totalmente dependiente. Patrón disfuncional.

\section{Patrón Valores-Creencias}

Practica la religión católica y juega un papel importante en su vida ya que le proporciona consuelo, fuerza y esperanza ante la situación actual; su mayor temor, que es morir, siempre está presente en estos días. Menciona que sus hijos y esposo le dan sentido a su vida y son su mayor preocupación en caso de fallecer. Patrón potencialmente disfuncional.

\section{Diagnósticos de enfermería (DE)}

Se integraron $13 \mathrm{DE}$, para estimar la precisión diagnóstica se empleó la EPDE de Lunney ${ }^{(19,20)}$, a través de la cual se seleccionaron ocho DE con precisión alta (figura 1) por ende se decidió diseñar el plan de cuidados y trabajar con estos ocho diagnósticos. La jerarquización de los DE con precisión alta, se estableció mediante el modelo AREA (21-23) (figura 2).

\section{Plan de cuidados}

Se presenta a continuación el plan de cuidados, en el que se incluyen los cinco DE que, de acuerdo a la EPDE de Lunney ${ }^{(19,20)}$ (figura 1 , columna 2 ) y a la jerarquización del modelo AREA (21-23) son relevantes para el estado de salud actual de la usuaria. Tres diagnósticos enfermeros que de igual forma son relevantes para la condición de la usuaria, no se incluyen en la propuesta por cuestiones de espacio. El plan de cuidados incluye, además, la evaluación de la respuesta de la usuaria al tratamiento de enfermería implementado. 


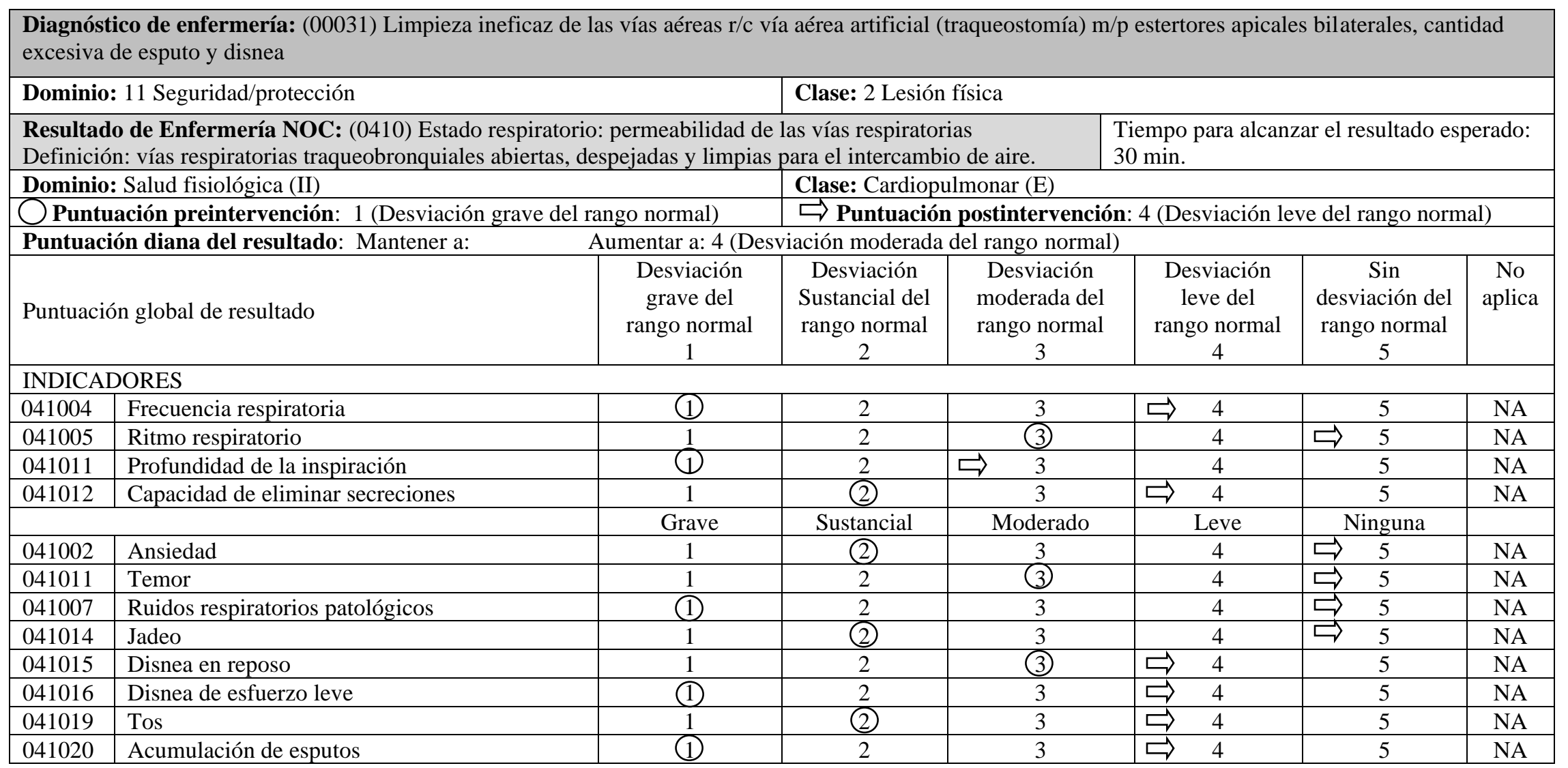


Intervención NIC: 3180 Manejo de las vías aéreas artificiales

Definición: Mantenimiento de tubos endotraqueales o de cánulas de traqueostomía y prevención de complicaciones asociadas con su utilización

Dominio: 2 Fisiológico: Complejo

\section{Actividades:}

- Realizar el lavado de manos.

- Emplear precauciones universales.

- Usar el equipo de protección personal.

- Proporcionar una humidificación del $100 \%$ al oxígeno inspirado.

- Proporcionar una hidratación sistémica adecuada mediante la administración oral e intravenosa.

- Comprobar cantidad y consistencia de las secreciones.

- Inflar el globo de traqueostomía mediante una técnica mínimamente oclusiva y de fugas mínimas.

- Mantener el inflado del globo de traqueostomía a 15-20mmHg durante la ventilación mecánica y durante y después de la alimentación.

- Comprobar la presión del globo de traqueostomía cada 4-8 horas durante la espiración mediante un manómetro.

- Comprobar la presión del globo inmediatamente después de manipulación.

- Realizar aspiración endotraqueal.

- Cambiar la sujeción de traqueostomía cada 24 horas, inspeccionar la piel y la mucosa bucal.

- Auscultar la presencia de sonidos pulmonares bilaterales después del cambio de sujeción de traqueostomía.

\section{Clase: K Control respiratorio}

- Observar si hay presencia de crepitaciones y roncus en las vías de gran calibre

- Monitorizar la disminución del volumen espirado y el aumento de la presión inspiratoria en los pacientes que reciben ventilación mecánica.

- Proporcionar cuidados a la tráquea cada 4-8 horas: limpiar cánula interna, limpiar y secar la zona periostomal y cambiar la sujeción de la traqueostomía.

- Inspeccionar y palpar para detectar la presencia de enfisema subcutáneo cada 8 horas.

- Realizar cuidados orales.

- Realizar una técnica estéril al succionar y proporcionar los cuidados de traqueostomía.

- Proteger la traqueostomía del agua.

- Realizar fisioterapia torácica.

- Elevar la cabecera de la cama $30^{\circ}$ o más.

\section{Fundamentación científica:}

Elizalde-González JJ, Fortuna-Custodio JA, Luviano-García JA, MendozaRomero VM. Guía COVID-19 para la atención del paciente crítico con infección por SARS-coV-2. Med Crítica [Internet]. 2020;33(1):7-42. ${ }^{(26)}$

\section{Evaluación:}

El resultado de las intervenciones de enfermería fue positivo ya que la usuaria pasó de permeabilidad de vías aéreas con desviación grave del rango normal (1), a desviación leve del rango normal (4). Por lo tanto, hay evidencia de un cambio positivo (+3), ya que la mayoría de las características definitorias desaparecieron y otras disminuyeron. 
Intervención NIC: 3350 Monitorización respiratoria

Definición: Recopilación y análisis de datos de un paciente para asegurar la permeabilidad de las vías aéreas y el intercambio gaseoso adecuado Dominio: 2 Fisiológico: Complejo

\section{Actividades:}

- Vigilar la frecuencia, ritmo, profundidad y esfuerzo de las respiraciones.

- Evaluar el movimiento torácico, observando la simetría, utilización de músculos accesorios y retracciones de músculos intercostales y supraclaviculares.

- Observar si se producen respiraciones ruidosas como estridor o ronquidos.

- Monitorizar los patrones de respiración.

- Monitorizar los niveles de saturación de oxígeno continuamente.

- Observar si hay fatiga muscular diafragmática.

- Auscultar los sonidos respiratorios, observando áreas de

disminución/ausencia de ventilación y presencia de sonidos adventicios.

- Determinar la necesidad de aspiración auscultando para ver si hay crepitación o roncus en las vías aéreas principales.

- Auscultar los sonidos pulmonares después del tratamiento para apreciar los resultados.

\section{Clase: K Control respiratorio}

- Monitorizar las lecturas del ventilador mecánico, anotando los aumentos de presiones inspiratorias y las disminuciones de volumen corriente.

- Monitorizar si aumenta la inquietud, ansiedad o disnea.

- Observar los cambios de $\mathrm{SaO} 2, \mathrm{SvO} 2$ y $\mathrm{CO} 2$ en valores de gasometría arterial.

- Comprobar la capacidad del paciente para toser eficazmente.

- Anortar la aparición, características y duración de la tos.

- Vigilarlas secreciones respiratorias del paciente.

- Observar si hay disnea y los factores que la mejorar y empeoran

- Monitorizar la presencia de crepitación.

Fundamentación científica:

Che-Morales JL, Díaz-Landero P, Cortés-Tellés A. Manejo integral del paciente con traqueostomía. Neumol Cir Torax [Internet]. 2014;73(4):25462. (27)

\section{Evaluación:}

El resultado de las intervenciones de enfermería fue positivo ya que la usuaria pasó de permeabilidad de vías aéreas con desviación grave del rango normal (1), a desviación leve del rango normal (4). Por lo tanto, hay evidencia de un cambio positivo (+3), demostrando así que la monitorización respiratoria es parte fundamental en el tratamiento de enfermería a pacientes con traqueostomía. 


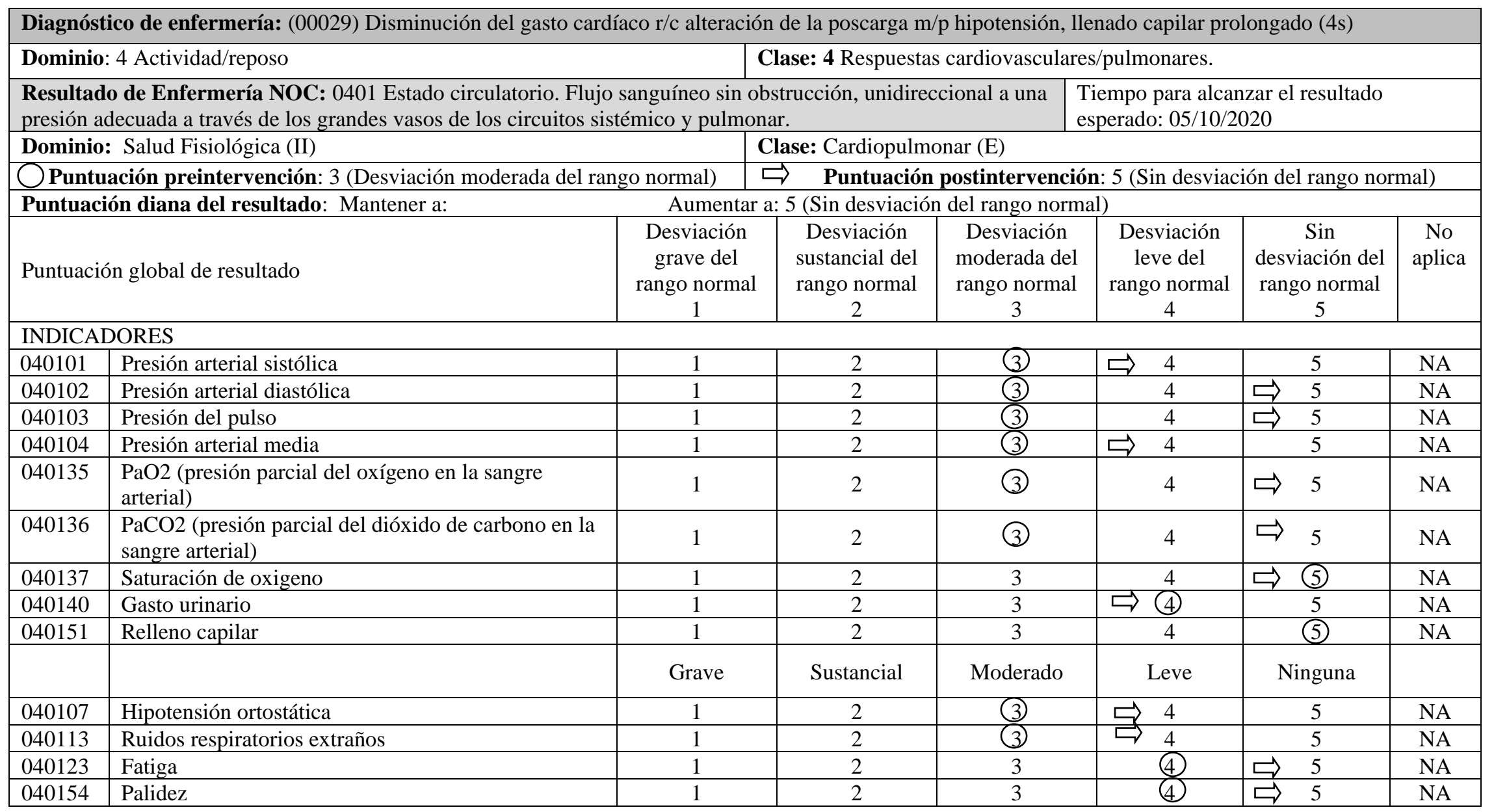




\section{Intervención NIC: 4150 Regulación hemodinámica}

Definición: Optimización de la frecuencia, la precarga, la poscarga y la contractilidad cardíacas.

Dominio: 2 Fisiológico: Complejo

\section{Actividades:}

- Realizar una evaluación exhaustiva del estado hemodinámico.

- Utilizar múltiples parámetros para determinar el estado clínico del paciente.

- Monitorizar y documentar la presión del pulso proporcional.

- Reconocer la presencia de signos y síntomas precoces de alerta indicativos de un compromiso en el sistema hemodinámico.

- Determinar el estado del volumen.

- Monitorizar los signos y síntomas de problemas del estado de volumen.

- Determinar el estado de perfusión.

- Monitorizar la presencia de signos y síntomas de problemas del estado de perfusión.

- Auscultar los sonidos pulmonares para ver si hay crepitantes u otros sonidos adventicios.

- Auscultar los ruidos cardíacos.

- Comprobar y registrar la presión arterial, la frecuencia, el ritmo cardíaco y pulsos.

- Administrar medicamentos vasoconstrictores.
Clase: $\mathbf{N}$ Control de la perfusión tisular

- Monitorizar los efectos de la medicación.

- Observar los pulsos periféricos, relleno capilar, la temperatura y el color de las extremidades.

- Elevar la cabecera de la cama.

- Monitorizar los niveles de electrolitos

- Comprobar y registrar la presión arterial, la frecuencia, el ritmo cardíaco y pulsos.

- Administrar medicamentos vasoconstrictores.

- Monitorizar los efectos de la medicación.

- Observar los pulsos periféricos, relleno capilar, la temperatura y el color de las extremidades.

- Elevar la cabecera de la cama.

- Monitorizar los niveles de electrolitos.

\section{Fundamentación científica:}

Freire ME, Llaguno RI, Semanate NM, Domínguez MF, Domínguez ND, Semanate SD. Manejo farmacológico del paciente hipotenso. Enfermería Investiga, Investigación, Vinculación, Docencia y Gestión. 2018;3(1 Sup):65-70. ${ }^{(28)}$

\section{Evaluación:}

El resultado de las intervenciones fue positivo, pues la usuaria pasó de un estado circulatorio con desviación moderada del rango normal (3) en la puntuación preintervención, a sin desviación del rango normal (5) en la puntuación postintervención. Existe evidencia de cambio positivo (+2), mejorando así el estado circulatorio de la usuaria y generando la desaparición de las características definitorias del diagnóstico de enfermería de la usuaria. 


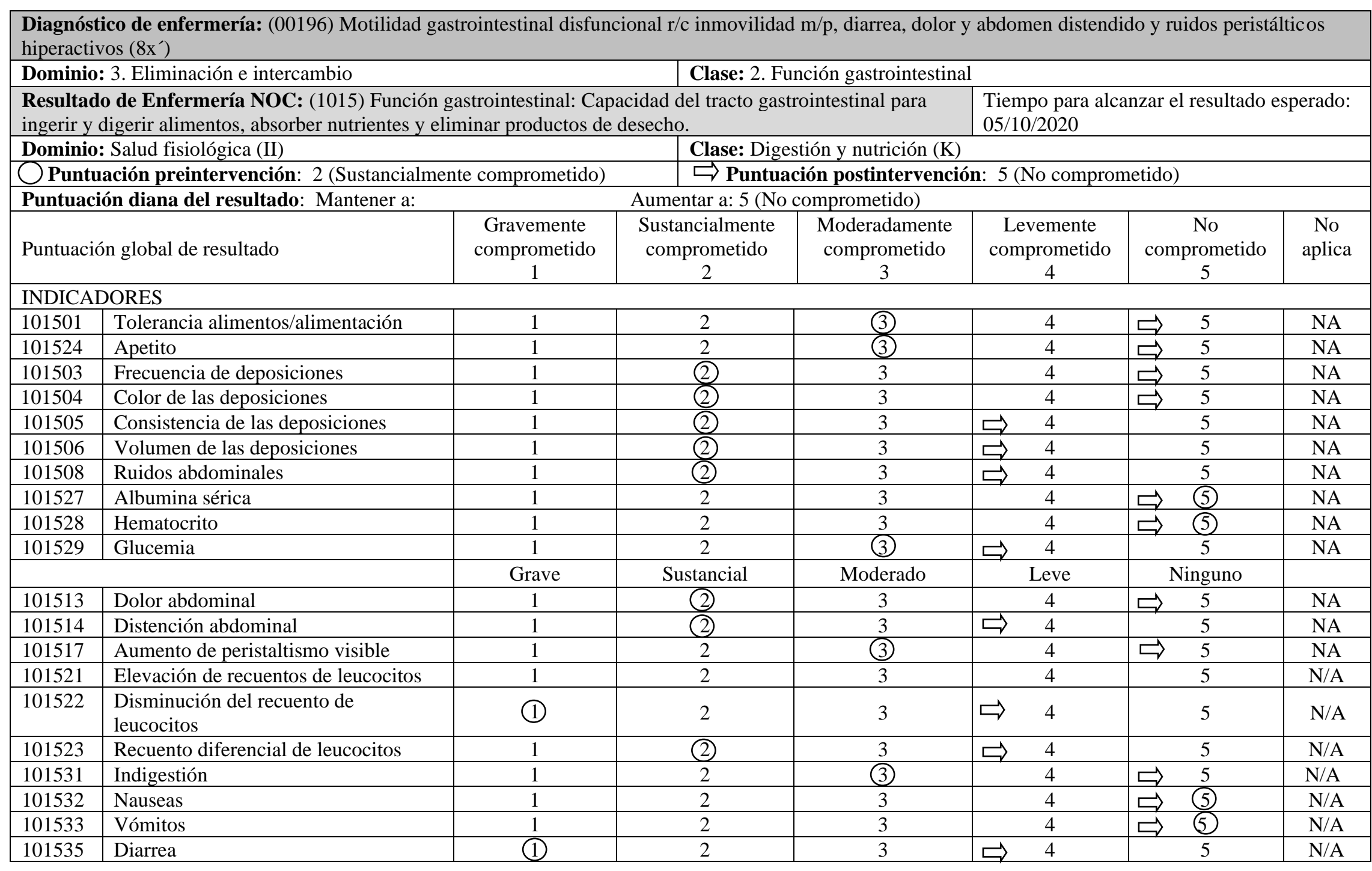


Intervención NIC: 0430 Control intestinal

Definición: Instauración y mantenimiento de un patrón regular de evacuación intestinal.

\begin{tabular}{l|l} 
Dominio: 1 Fisiológico: Básico & Clase: B Control de la eliminación
\end{tabular}

\begin{tabular}{l|l} 
Actividades: & $\bullet$ Evaluar la incontinencia fecal.
\end{tabular}

- Anotar la fecha de la última defecación.

- Monitorización las defecaciones, incluyendo la frecuencia, consistencia, forma, volumen y color.

- Monitorizar los sonidos intestinales.

- Informar acerca de cualquier aumento frecuencia y/o sonidos intestinales agudos.

- Informar si hay disminución de los sonidos intestinales.

- Monitorizar los signos y síntomas de diarrea, estreñimiento e impactación.

- Tomar nota de problemas intestinales, rutina intestinal y uso de laxantes previos.

\section{Evaluación:}

El resultado de la intervención fue positivo ya que se instauró la función gastrointestinal al pasar de sustancialmente comprometido (2) en la puntuación preintervención, a no comprometido (5) en la puntuación post intervención. Hay evidencia de cambio positivo (+3), ya que las características definitorias del diagnóstico de enfermería que presentaba la usuaria disminuyeron o desaparecieron por completo. 


\begin{tabular}{|c|c|c|c|c|c|c|c|}
\hline \multicolumn{8}{|c|}{ gnóstico de enfermería: (00155) Riesgo de caídas c/d deterioro de la movilidad, alteración del nivel de glucosa en sangre (tendencia a la hiperglucemia) } \\
\hline \multicolumn{5}{|c|}{$\begin{array}{l}\text { Resultado de Enfermería NOC: (1909) Conducta de prevención de caídas: Acciones personales o del } \\
\text { cuidador familiar para minimizar los factores de riesgo que podrían producir caídas en el entorno personal }\end{array}$} & \multicolumn{3}{|c|}{$\begin{array}{l}\text { Tiempo para alcanzar el resultado esperado: } \\
05 / 10 / 2020\end{array}$} \\
\hline \multicolumn{3}{|c|}{ Dominio: Conocimiento y conducta de salud (IV) } & \multicolumn{5}{|c|}{ Clase: Seguridad $(\mathrm{HH})$} \\
\hline \multicolumn{3}{|c|}{ Puntuación preintervención: 3 (A veces demostrado) } & \multicolumn{5}{|c|}{$\Rightarrow$ Puntuación postintervención: 4 (Frecuentemente demostrado) } \\
\hline \multicolumn{8}{|c|}{ Puntuación diana del resultado: Mantener a: $\quad$ Aumentar a: 4 (Frecuentemente demostrado) } \\
\hline \multicolumn{2}{|c|}{ Puntuación global de resultado } & $\begin{array}{c}\text { Nunca } \\
\text { demostrado } \\
1\end{array}$ & $\begin{array}{c}\text { Raramente } \\
\text { demostrado } \\
2\end{array}$ & $\begin{array}{c}\text { A veces } \\
\text { demostrado } \\
3\end{array}$ & $\begin{array}{c}\text { Frecuentemente } \\
\text { demostrado } \\
4 \\
\end{array}$ & $\begin{array}{c}\text { Siempre } \\
\text { demostrado } \\
5\end{array}$ & No aplica \\
\hline \multicolumn{8}{|c|}{ INDICADORES } \\
\hline 190923 & Pide ayuda & 1 & 2 & 3 & (4) & 5 & NA \\
\hline 190903 & Coloca barreras para prevenir caídas & 1 & 2 & 3 & (4) & $\Rightarrow$ & NA \\
\hline 190901 & Utiliza dispositivos de ayuda correctamente & 1 & 2 & (3) & $\Rightarrow$ & 5 & NA \\
\hline 190918 & Utiliza dispositivos oculares de corrección & 1 & 2 & (3) & 4 & 5 & NA \\
\hline 190902 & Proporciona ayuda para la movilidad & 1 & 2 & (3) & $\Rightarrow$ & 5 & NA \\
\hline 190919 & Utiliza procedimientos de traslado seguros & 1 & 2 & (3) & 4 & $\Rightarrow$ & NA \\
\hline 190913 & Adapta la altura de la cama según sea necesario & 1 & 2 & 3 & (4) & $\Rightarrow$ & NA \\
\hline 190916 & Controla la inquietud & 1 & (2) & 3 & $\Rightarrow$ & 5 & NA \\
\hline 190917 & $\begin{array}{l}\text { Utiliza precauciones cuando toma medicamentos que } \\
\text { aumentan el riesgo de caídas }\end{array}$ & (1) & 2 & 3 & $\Rightarrow$ & 5 & NA \\
\hline
\end{tabular}


Intervención NIC: 6490 Prevención de caídas

Definición: Establecer precauciones especiales en pacientes con alto riesgo de lesiones por caídas.

\section{Actividades:}

- Identificar déficits cognitivos o físicos del paciente que puedan aumentar la posibilidad de caídas en un ambiente dado.

- Identificar conductas y factores que afectan al riesgo de caídas.

- Revisar los antecedentes de caídas con el paciente y la familia.

- Identificar las características del ambiente que puedan aumentar las posibilidades de caídas.

- Bloquear las ruedas de la cama y sillón

- Evite reorganizaciones innecesarias del ambiente físico.

\section{Dominio: 4 Seguridad}

\section{Clase: V Control de riesgos}

- Colocar la cama mecánica en la posición más baja

- Proporcionar al paciente dependiente de medios de solicitud de ayuda.

- Ayudar en el aseo en intervalos frecuentes.

\section{Fundamentación científica:}

Khan MSZ, Zaman AKA, Doza AAU. Physical Rehabilitation in the ICU.

Bangladesh Crit Care J. 2015;3(2):63-6. ${ }^{(30}$

Hernández-Herrera D, Aguilera-Elizarraraz N, Vega-Argote M, González-Quirarte

N, Castañeda-Hidalgo H, Isasi-Hernández L. Aplicación de las actividades de la

intervención de enfermería Prevención de caídas en adultos hospitalizados.

Enfermería Univ. 2017:14(2):118-23. (31)

\section{Evaluación:}

El resultado de las intervenciones fue positivo ya que comparando la puntuación previa a las intervenciones que fue de a veces demostrado (3), con la

puntuación postintervención que fue frecuentemente demostrado (4), existe cambio de +1 , lo que demuestra que la usuaria adquirió una conducta de prevención de caídas gracias a las actividades realizadas. 
Intervención NIC: 0226 Terapia de ejercicios: control muscular

Definición: Utilización de protocolos de actividad o ejercicios específicos para mejorar o restablecer el movimiento controlado del cuerpo

Dominio: 1 Fisiológico: Básico

\section{Actividades:}

- Determinar la disposición del paciente para comprometerse a realizar un protocolo de actividades o ejercicios.

- Colaborar con fisioterapeutas, terapeutas ocupacionales y recreacionales en el desarrollo y ejecución de un programa de ejercicios.

- Consultar con el fisioterapeuta para determinar la posición óptima del paciente durante el ejercicio y el número de veces que debe realizar cada patrón de movimiento.

- Evaluar las funciones sensoriales.

- Explicar el fundamento del tipo de ejercicio y el protocolo al paciente.

- Establecer una secuencia de actividades diarias cuidados para potenciar los efectos de la terapia específica de ejercicios.

- Poner en marcha medidas del control de dolor antes de comenzar el ejercicio/actividad.

- Ayudar a mantener la estabilidad del tronco y/o articulaciones proximales implicadas en las habilidades motoras finas.

\section{Evaluación:}

El resultado de las intervenciones fue positivo ya que comparando la puntuación previa a las intervenciones que fue de a veces demostrado (3), con la puntuación postintervención que fue frecuentemente demostrado (4), existe un avance de +1 ; logrando así la disminución de la vulnerabilidad en la usuaria para presentar una caída o lesión debido a su deterioro de la movilidad.
- Determinar la precisión de la imagen corporal.

- Proporcionar instrucciones secuenciales para cada actividad motora durante el ejercicio.

- Proporcionar un ambiente relajado al paciente después de cada periodo de ejercicios.

- Ayudar al paciente a desarrollar el protocolo de ejercicios para conseguir resistencia, fortaleza y flexibilidad.

- Utilizar estímulos táctiles para minimizar el espasmo muscular.

- Vigilar la respuesta emocional, cardiovascular y funcional del paciente al protocolo de ejercicios.

\section{Fundamentación científica:}

Villamil P, Hernández Á, Moscoso L. Eficacia del ejercicio físico terapéutico en pacientes adultos hospitalizados en UCI: revisión sistemática y metaanálisis.

Fisioterapia. 2020; 42, (2): 98-107. ${ }^{(32)}$ 
Intervención NIC: 2120 Manejo de la hiperglucemia

Definición: Prevenir y tratar los niveles sanguíneos de glucemia superiores a lo normal

Dominio: 2 Fisiológico: Complejo

Actividades:

- Vigilar la glucemia.

- Observar si hay signos y síntomas de hiperglucemia: poliuria, polidipsia, polifagia, debilidad, malestar, letargo, visión borrosa o cefalea.

- Vigilar la presencia de cuerpos cetónicos en orina.

- Comprobar la gasometría arterial y los niveles de electrolitos.

- Monitorizar la presión arterial y el pulso.

- Administrar insulina.

- Potenciar la ingesta oral de líquidos.

- Monitorizar el balance hídrico.

Clase: G Control de electrólitos y acido básico

- Mantener una línea I.V.

- Administrar líquidos I.V.

- Facilitar la higiene bucal.

- Identificar las causas posibles de la hiperglucemia.

- Anticiparse a las situaciones en las que aumentarán las necesidades de insulina.

\section{Fundamentación científica:}

Martínez-Gangoso L, Fuentes-Pumarola C. La hiperglucemia en el

paciente crítico. Vía de elección, controles y valores: Revisión

bibliográfica. Enferm Intensiva. 2014;25(4):146-63. ${ }^{(33)}$

\section{Evaluación:}

El resultado de las intervenciones fue positivo ya que comparando la puntuación previa a las intervenciones que fue de a veces demostrado (3), con la

puntuación postintervención que fue frecuentemente demostrado (4), existe un avance de +1 ; esto muestra que, al controlar las cifras de glucosa en sangre, se

disminuye el riesgo de caídas. 
Figura 1. Escala de Precisión de Diagnósticos de Enfermería

\begin{tabular}{|c|c|c|c|c|c|c|c|c|c|c|c|c|}
\hline \multirow{2}{*}{$\begin{array}{l}\text { DIAGNÓSTICOS DE } \\
\text { ENFERMERÍA }\end{array}$} & \multicolumn{2}{|c|}{$\begin{array}{l}\text { Presencia } \\
\text { de datos }\end{array}$} & \multicolumn{2}{|c|}{$\begin{array}{c}\text { Relevancia de los } \\
\text { datos }\end{array}$} & \multicolumn{2}{|c|}{$\begin{array}{c}\text { Especificidad de } \\
\text { los datos }\end{array}$} & \multicolumn{2}{|c|}{$\begin{array}{c}\text { Coherencia de los } \\
\text { datos }\end{array}$} & \multicolumn{2}{|c|}{$\begin{array}{l}\text { ¿Mantiene el } \\
\text { diagnóstico? }\end{array}$} & \multirow{2}{*}{$\begin{array}{l}\text { PUNTUACIÓN } \\
\text { DE LA } \\
\text { PRECISIÓN }\end{array}$} & \multirow{2}{*}{$\begin{array}{l}\text { CATEGORÍA } \\
\text { DE } \\
\text { PRECISIÓN** }\end{array}$} \\
\hline & $\begin{array}{l}\mathrm{Si} \\
(1)\end{array}$ & $\begin{array}{l}\text { No } \\
(0)\end{array}$ & $\begin{array}{l}\text { Alto/ } \\
\text { Moderado } \\
(1) \\
\end{array}$ & $\begin{array}{c}\text { Bajo } \\
(0)\end{array}$ & $\begin{array}{c}\text { Alto/ } \\
\text { Moderado } \\
(1) \\
\end{array}$ & $\begin{array}{c}\text { Bajo } \\
(0)\end{array}$ & $\begin{array}{l}\text { Alto/ } \\
\text { Moderado } \\
(1) \\
\end{array}$ & $\begin{array}{c}\text { Bajo } \\
(0)\end{array}$ & $\mathrm{Si}$ & No & & \\
\hline $\begin{array}{l}\text { (00030) Deterioro del intercambio de gases } \\
\text { r/c desequilibrio en la ventilación-perfusión } \\
\text { m/p gasometría arterial anormal (acidosis } \\
\text { metabólica medianamente compensada, } \\
\text { índice de Kirby de 196) }\end{array}$ & 1 & & 1 & & 3.5 & & 8 & & SI & & 13.5 & ALTA \\
\hline $\begin{array}{l}\text { (00031) Limpieza ineficaz de las vías aéreas } \\
\text { r/c vía aérea artificial (traqueostomía) m/p } \\
\text { estertores apicales bilaterales, cantidad } \\
\text { excesiva de esputo y disnea }\end{array}$ & 1 & & 1 & & 3.5 & & 8 & & SI & & 13.5 & ALTA \\
\hline $\begin{array}{l}\text { (00029) Disminución del gasto cardíaco r/c } \\
\text { alteración de la poscarga } \mathrm{m} / \mathrm{p} \text { hipotensión, } \\
\text { Ilenado capilar prolongado }(4 \mathrm{~s})\end{array}$ & 1 & & 1 & & & 0 & 8 & & SI & & 10 & ALTA \\
\hline $\begin{array}{l}\text { (00155) Riesgo de caídas c/d deterioro de la } \\
\text { movilidad, alteración del nivel de glucosa en } \\
\text { sangre (tendencia a la hiperglucemia), } \\
\text { diarrea }\end{array}$ & 1 & & 1 & & 3.5 & & 8 & & SI & & 13.5 & ALTA \\
\hline $\begin{array}{l}\text { (0046) Deterioro de la integridad cutánea r/c } \\
\text { inmovilidad física, presión y cizallamiento y } \\
\text { diarrea } \mathrm{m} / \mathrm{p} \text { lesión por dependencia de tipo } \\
\text { mixto grado II en región inter glútea y dolor } \\
\text { (8 en EVA) }\end{array}$ & 1 & & 1 & & 3.5 & & 8 & & SI & & 13.5 & ALTA \\
\hline $\begin{array}{l}\text { (00196) Motilidad gastrointestinal } \\
\text { disfuncional } r / c \text { inmovilidad } \mathrm{m} / \mathrm{p} \text {, diarrea, } \\
\text { dolor y abdomen distendido y ruidos } \\
\text { peristálticos hiperactivos }\left(8 \mathrm{x}^{\prime}\right)\end{array}$ & 1 & & 1 & & 3.5 & & 8 & & SI & & 13.5 & ALTA \\
\hline $\begin{array}{l}\text { (00048) Deterioro de la dentición } \mathrm{r} / \mathrm{c} \text { acceso } \\
\text { difícil al cuidado dental, higiene oral } \\
\text { inadecuada } \mathrm{m} / \mathrm{p} \text { sarro y caries, halitosis y } \\
\text { anodoncia parcial }\end{array}$ & 1 & & & 0 & 3.5 & & 8 & & SI & & 12.5 & ALTA \\
\hline $\begin{array}{l}\text { (00232) Obesidad r/c actividad física diaria } \\
\text { menor a la recomendada y gasto energético } \\
\text { menor al consumo de energía m/p IMC del } \\
30 \mathrm{~kg} / \mathrm{m}^{2}\end{array}$ & 1 & & & 0 & 3.5 & & 8 & & SI & & 12.5 & ALTA \\
\hline
\end{tabular}




\begin{tabular}{|c|c|c|c|c|c|c|c|}
\hline $\begin{array}{l}\text { (00195) Riesgo de desequilibrio electrolítico } \\
\text { r/c diarrea, mecanismos de regulación } \\
\text { comprometidos y volumen de líquidos } \\
\text { insuficiente }\end{array}$ & 1 & 0 & 0 & 0 & NO & 1 & BAJA \\
\hline $\begin{array}{l}\text { (00034) Respuesta ventilatoria disfuncional } \\
\text { al destete } r / c \text { antecedentes de dependencia } \\
\text { del ventilador mayor a } 4 \text { días } m / p \text { aumento } \\
\text { moderado de la frecuencia respiratoria sobre } \\
\text { la basal }\left(36 x^{\prime}\right) \text {. }\end{array}$ & 1 & 0 & 0 & 0 & NO & 1 & BAJA \\
\hline $\begin{array}{l}\text { (00043) Protección ineficaz r/c perfil } \\
\text { hematológico anormal } \mathrm{m} / \mathrm{p} \text { anemia y } \\
\text { leucopenia. }\end{array}$ & 1 & 0 & 0 & 0 & NO & 1 & BAJA \\
\hline $\begin{array}{l}\text { (00092) Intolerancia a la actividad } \mathrm{r} / \mathrm{c} \\
\text { desequilibrio entre aporte y demanda de } \\
\text { oxígeno } \mathrm{m} / \mathrm{p} \text { disnea de esfuerzo y fatiga. }\end{array}$ & 1 & 0 & 0 & 0 & NO & 1 & BAJA \\
\hline $\begin{array}{l}\text { (00203) Riesgo de perfusión renal ineficaz } \\
\mathrm{r} / \mathrm{c} \text { alteración del metabolismo (urea } 57.8 \\
\mathrm{mg} / \mathrm{dL} \text { ). }\end{array}$ & 1 & 0 & 0 & 0 & NO & 1 & BAJA \\
\hline
\end{tabular}

Fuente: Elaboración propia. 
Figura 2. Red de razonamiento clínico

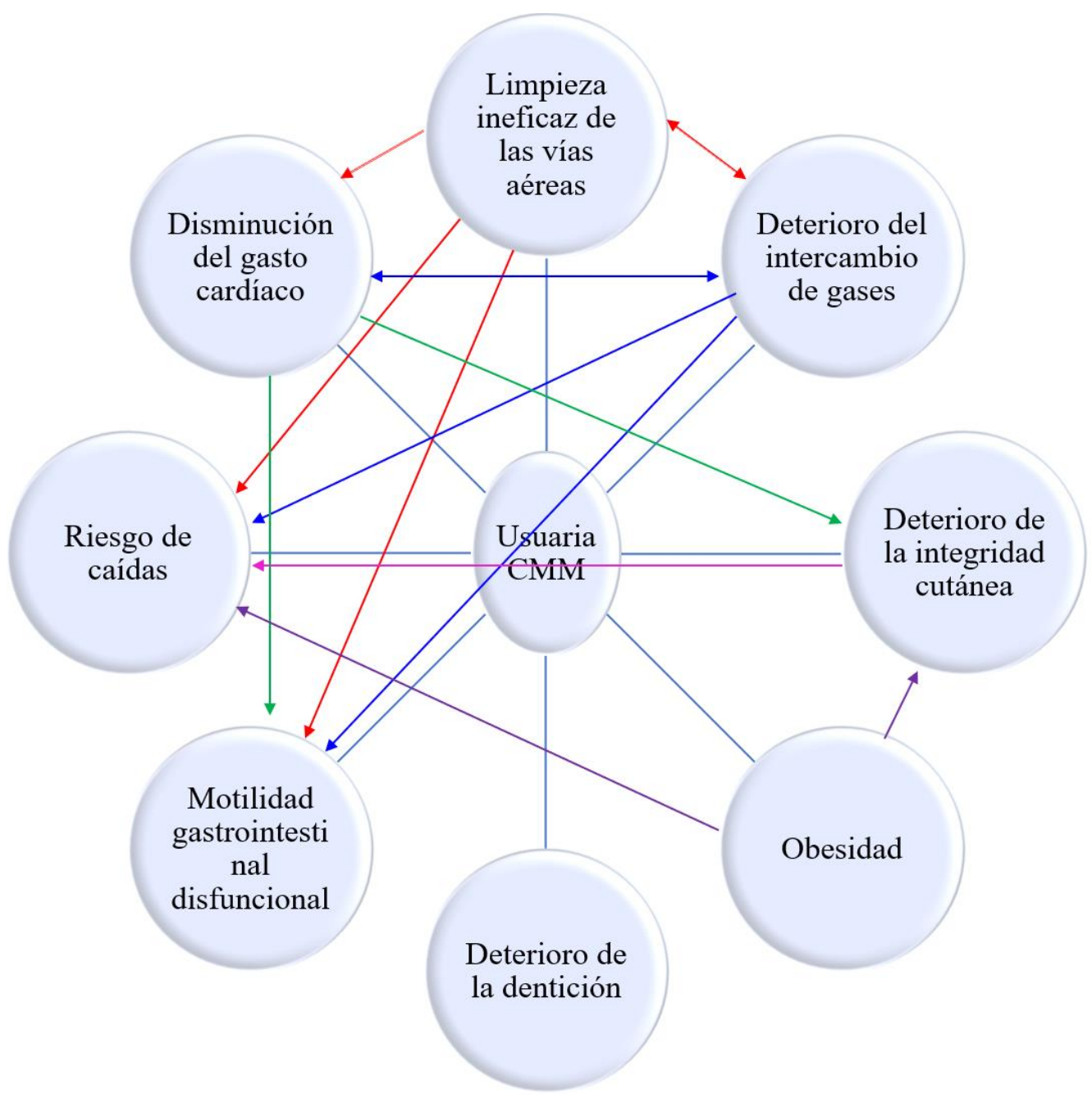

Fuente: Elaboración propia.

\section{CONCLUSIONES}

La implementación del cuidado profesional de enfermería requiere el sustento de métodos y herramientas propias de la disciplina. El cuidado enfermero de la usuaria CMM, se sustentó en el proceso de enfermería, las taxonomías enfermeras NANDA, NOC y NIC, y dos herramientas que fomentan el juicio clínico enfermero: la Escala de Precisión de Diagnósticos de Enfermería que, a través del juicio diagnóstico permitió valorar la precisión de los diagnósticos enfermeros; y el modelo AREA, que facilitó la identificación de diagnósticos principales y secundarios. También se puso en práctica el juicio terapéutico en la elección del tratamiento de enfermería.

La implicación del pensamiento crítico en el quehacer enfermero es inherente, así mismo, la aplicación de métodos y herramientas propias de la disciplina es evidencia de los procesos 
intelectuales que se reflejan en las intervenciones de enfermería, fomentando indudablemente la autonomía de la disciplina enfermera.

La usuaria CMM egresó por mejoría y expresó bienestar y satisfacción por el cuidado de enfermería recibido. La participación del profesional de enfermería en el tratamiento de usuarios diagnosticados con COVID-19, es esencial dado que es la enfermera quien identifica tempranamente los cambios en el estado de salud y los monitorea, implementa los tratamientos específicos y evalúa la respuesta a éstos.

\section{CONFLICTO DE INTERESES}

Las autoras declaran no tener conflicto de intereses.

\section{FINANCIAMIENTO}

Ninguno.

\section{REFERENCIAS BIBLIOGRÁFICAS}

1. Cadena-Estrada JC, Olvera-Arreola SS, López-Flores L, et al. Enfermería ante el COVID-19, un punto clave para la prevención, control y mitigación de la pandemia. Cardiovasc Metab Sci. [Internet]. 2020 [Consultado en octubre 2020]; 31(Suppl:3):265-270. Disponible en: https://doi.org/10.35366/93958

2. Secretaría de Salud. Informe Técnico Diario COVID-19 MÉXICO [Internet]. Subsecretaría de Prevención y Promoción de la Salud; 2020. [Consultado en octubre 2020]. Disponible en: https://www.gob.mx/cms/uploads/attachme nt/file/587674/Comunicado_Tecnico_Diario_ COVID-19_2020.10.24.pdf

3. Álvarez-López DI, Espinoza-Molina MP, CruzLoustaunau ID, et al. La diabetes e hipertensión arterial como factores asociados con la letalidad por Covid-19 en Sonora, México. Salud Pública de México[Internet]. 2020. [Consultado en octubre 2020]. 62(5): 456-457. Disponible en: https://doi.org/10.21149/11546

4. Morales B, Palencia J. Reflexiones del cuidado enfermero en tiempos de COVID-19. Enfermería Investiga, Investigación, Vinculación, Docencia y Gestión[Internet].2020. [Consultado octubre 2020] 5(3): 71-78. Disponible en: https://revistas.uta.edu.ec/erevista/index.ph p/enfi/article/view/914/859

5. Canova-Barrios C, Salvador S. Cuidados de Enfermería en la infección por COVID-19: y entonces, ¿cómo procedemos? Cuidados de Enfermería y Educación en Salud. [Internet]. 2020 [Consultado octubre 2020] 5(1): 37-51. Disponible

en: https://revistas.userena.cl/index.php/cuidenf /article/view/1256/1350

6. Andrade R, López J, Venegas M. Proceso de atención de enfermería. Libro de Texto. $1^{\text {a }}$ ed. México: Trillas; 2018.

7. Alfaro-Lefevre R. Pensamiento crítico y juicio clínico en enfermería. $6^{a}$ ed. España: Elsevier; 2017.

8. Alfaro-Lefevre R. Aplicación del proceso enfermero. Fundamento del razonamiento clínico. $8^{\mathrm{a}}$ ed. España: Editorial Lippincott; 2014.

9. Gordon M. Diagnóstico Enfermero. Proceso y Aplicación. $3^{a}$ ed. España: Ed. Mosby; 1996.

10. Weber J. Manual de valoración de la salud en enfermería. $1^{\text {a }}$ ed. España: Lippincott; 2018. 
11. Herdman TH, Kamitsuru S. NANDAInternational. Diagnósticos enfermeros. Definiciones y clasificación 2018-2020. España: Elsevier; 2017.

12. Luis R. Los diagnósticos enfermeros. Revisión crítica y guía práctica. $9^{a}$ ed. España: Elsevier Masson; 2013.

13. Luis R. Enfermería Clínica. Cuidados enfermeros a las personas con trastornos de la salud. $1^{\mathrm{a}}$ ed. España: LWW; 2015.

14. Wilkinson J. Manual de diagnósticos de enfermería. 10a ed. España: Pearson; 2016.

15. Moorhead S, Swanson E, Johnson M, Mass M. Clasificación de Resultados de Enfermería (NOC). 6th ed. España: Elsevier; 2018.

16. Rifa R., Olivé C. y Lamoglia M. Lenguaje NIC para el aprendizaje teórico-práctico en enfermería. $2^{a}$ ed. España: Elsevier; 2020.

17. Butcher $H$, Bulechek $G$, Dochterman J, Wagner Ch. Clasificación de Intervenciones de Enfermería (NIC). 7th ed. España: Elsevier; 2018.

18. Herramienta online para la consulta y diseño de Planes de Cuidados de Enfermería. [Internet]. NNNConsult. Elsevier; 2015. [Consultado septiembre 2020]. Disponible en: http://www.nnnconsult.com/

19. Lunney M. Razonamiento crítico para alcanzar resultados de salud positivos. Estudios de casos y análisis de enfermería. $1^{\text {a }}$ ed. España: Elsevier; 2011.

20. Rifá $R$, Rodríguez $M$, Pérez I, Lamoglia $M$, Costa L. Relación entre precisión diagnóstica y actitud frente a los diagnósticos de enfermería en estudiantes de pregrado. Educación Médica. [Internet]. 2019
[Consultado septiembre 2020]; 20(S2): 33-38. Disponible

en: https://doi.org/10.1016/j.edumed.2017.12.0 07

21. Kuiper R, Pesut D, Arms T. Clinical Reasoning and Care Coordination in Advanced Practice Nursing. $1^{\text {a }}$ ed. New York: Springer [Internet]; 2016. [Consultado octubre 2020]. Disponible en:http://web.a.ebscohost.com/ehost/ebook viewer/ebook/ZTAwMHh3d19fMTIzMzkyOF 9fQU41?sid=8708f6ca-56e2-4272-be2bed419547b24c@sdc-vsessmgr01\&vid $=2 \&$ format $=E B \&$ rid $=1$

22. Ibáñez-Alfonso LE, Fajardo-Peña $M T$, Cardozo-Ortiz CE, Roa-Díaz ZM. Planes de cuidados enfermeros de estudiantes de pregrado: comparación de dos modelos. Salud UIS [Internet]. 2020 [Consultado octubre 2020]; 52(1): 33-40. Disponible en: http://dx.doi.org/10.18273/revsal.v52n12020005

23. Wuryanto E, Rahayu GR, Emilia O, Harsono O, Octavia AP. Application of an outcome present test-peer learning model to improve clinical reasoning of nursing students in the intensive care unit. Ann of Trop Med Public Health [Internet]. 2017 [Consultado octubre 2020]; 10(3):657-663. Disponible en: https://www.atmph.org/text.asp?2017/10/3/ $657 / 213137$

24. García-Fernández FP, Pancorbo-Hidalgo PL, Verdú-Soriano J, López-Casanova $P$, Rodríguez-Palma M. Documento Técnico GNEAUPP No II "Clasificación-categorización de las lesiones relacionadas con la dependencia" [Internet]. 2da. Grupo Nacional para el Estudio y Asesoramiento en Úlceras por presión y Heridas Crónicas. España: Grupo nacional para el estudio y asesoramiento en úlceras por presión y heridas crónicas; 2014 [Consultado octubre 2020]. 1-50 p. Disponible en: 
https://gneaupp.info/clasificacion-

categorizacion-de-las-lesiones-

relacionadas-con-la-dependencia-2/

25. Hinkle JL, Cheever $\mathrm{KH}$, et al. Enfermería médicoquirúrgica de Brunner y Suddarth $14 .^{a}$ ed. Barcelona, España: Wolters Kluwer; 2019. 13. Líquidos y electrolitos. Alteraciones acido básicas $286 \mathrm{p}$.

26. Elizalde-González JJ, Fortuna-Custodio JA, Luviano-García JA, Mendoza-Romero VM. Guía COVID-19 para la atención del paciente crítico con infección por SARS-coV-2 Colegio Mexicano de Medicina Crítica. Med Crítica [Internet]. 2020 [Consultado octubre 2020]; 33(1):7-42.

Disponible en: https://www.medigraphic.com/pdfs/medcri/ ti-2020/ti201b.pdf
27. Che-Morales JL, Díaz-Landero P, CortésTellés A. Manejo integral del paciente con traqueostomía. Neumol Cir Torax [Internet]. 2014 [Consultado octubre 2020];73(4):25462. Disponible en: https://www.medigraphic.com/pdfs/neumo/ nt-2014/nt144f.pdf

28. Freire $M E$, Llaguno $R I$, Semanate $N M$, Domínguez MF, Domínguez ND, Semanate SD. Manejo farmacológico del paciente hipotenso. Enfermería Investiga, Investigación, Vinculación, Docencia y Gestión. [Internet]. 2018 [Consulta octubre 2020];3(1 Sup):65-70. Disponible en: http://dx.doi.org/10.29033/ei.v3sup1.2018.1 4

Copyright (C) 2020 SANUS Artículo de acceso abierto distribuido bajo Licencia Creative Commons 